\title{
Hippocampal Lesions Disrupt Decrements but Not Increments in Conditioned Stimulus Processing
}

\author{
Jung-Soo Han, ${ }^{1}$ Michela Gallagher, ${ }^{1}$ and Peter Holland ${ }^{2}$ \\ 'Department of Psychology, University of North Carolina at Chapel Hill, Chapel Hill, North Carolina 27599 and \\ ${ }^{2}$ Department of Psychology, Duke University, Durham, North Carolina 27706
}

\begin{abstract}
Studies recording hippocampal neural activity show widespread registration of events during associative learning. Recent computational models of hippocampal function have stressed its role in attentional processes specified by well-developed modern theories of associative learning. These modeling efforts are largely aimed at accounting for the behavioral outcomes of damage to the hippocampal system in terms of underlying changes in information processing. Two experiments examined the effects of neurotoxic lesions of the hippocampus on changes in attentional processing of a conditioned stimulus (CS) in appetitive Pavlovian conditioning in rats. In Experiment 1, hippocampal lesions eliminated the reduction in associability of a CS usually produced by preexposure to that cue (latent inhibition). In Experiment 2, hippocampal lesions interfered with the loss in associability of a CS normally produced when that CS consistently predicts another event. In contrast, in Experiment 2, hippocampal lesions did not prevent the enhancement of CS associability when a previously consistent predictive relation between two events was made inconsistent. This research supports previous claims that the hippocampus is involved in regulating the processing of CSs in Pavlovian conditioning, and provides new evidence for a hippocampal role in decremental, but not incremental, changes in attention.
\end{abstract}

[Key words: hippocampus, dentate gyrus, classical conditioning, attention, latent inhibition, ibotenic acid, rats]

Many studies have demonstrated learning-dependent neuronal processes in the hippocampus during classical conditioning. A conditioned stimulus (CS), such as a light or tone, that signals the occurrence of reinforcement comes to activate neurons throughout the hippocampus (e.g., Olds et al., 1972; Segal et al., 1972; Berger et al., 1976), as well as to elicit behavioral conditioned responses (CRs). Despite a detailed hippocampal registration of events in classical conditioning, however, the results of many lesion studies indicate that an intact hippocampal formation is not essential to the acquisition of CRs in many classical conditioning procedures (e.g., Schmaltz and Theios, 1972; Solomon and Moore, 1975; Berger and Orr, 1983, 1985).

\footnotetext{
Received Apr. 10, 1995; revised July 3, 1995; accepted July 6, 1995.

This research was supported by grants from the National Institute of Mental Health (KO5-MHO1149) and the Human Frontier Science Research Program to P.C.H. and M.G. We thank P. N. Fielder for technical assistance.

Correspondence should be addressed to Michela Gallagher, Department of Psychology, Davie Hall, CB\# 3270, The University of North Carolina, Chapel Hill, NC 27599

Copyright $\bigcirc 1995$ Society for Neuroscience $0270-6474 / 95 / 157323-07 \$ 05.00 / 0$
}

In contrast, there is ample evidence that the hippocampal formation is importantly involved in changes in the salience or associability of cues in conditioning paradigms. For example, in the latent inhibition procedure, repeated preexposure to a cue normally slows conditioning to that cue when it is subsequently paired with a reinforcer. This loss of CS salience in latent inhibition is reflected in reduced hippocampal neuronal responses to the preexposed cue in intact animals (Best and Best, 1976). Moreover, in animals with hippocampal damage produced by aspiration or electrolytic lesions, latent inhibition is substantially reduced, that is, subsequent conditioning of a preexposed stimulus is not retarded (e.g., Kaye and Pearce, 1987a,b; Solomon and Moore, 1975).

These findings are all consistent with early views that the hippocampus is involved in learning to ignore unimportant events (e.g. Douglas, 1967). On the whole, however, those early approaches failed to specify the conditions under which such filtering would take place. More recent, computational theories (c.g., Schmajuk and Moorc, 1988; Schmajuk and DiCarlo, 1991; Gluck and Meyers, 1993) have attempted to describe hippocampal function in the context of attentional processes postulated within well-developed modern theories of associative learning. Those theories specify rules by which attentional processing of conditioned stimuli (CSs) is altered, and how those alterations affect subsequent learning (see Holland and Gallagher, 1995, for a recent review).

Interestingly, whereas behavioral learning theories generally specify both the conditions that reduce attention and those that increase attention, studies of the hippocampus have been limited to situations in which reduced attention occurs in intact subjects. Although many investigators have implicated hippocampal circuitry in decremental changes in CS processing (e.g., Solomon and Moore, 1975; Rickert et al., 1978; Kaye and Pearce, 1987a,b), there has been no investigation of the involvement of the hippocampus when conditioning procedures that augment the salience of cues are used. The present experiments examined the effects of selective neurotoxic lesions of the hippocampus and dentate gyrus on both increases and decreases in CS salience induced by conditioning procedures. Latent inhibition was studied in Experiment 1. Experiment 2 examined performance in a serial conditioning task that elsewhere has been found to produce both decremental and incremental changes in CS processing (Wilson et al., 1992; Holland and Gallagher, 1993a).

\section{Experiment 1}

In Experiment 1, all rats received initial nonreinforced preexposure to one of two visual CSs. Then, both visual CSs were individually paired with food. Slower acquisition of conditioned 


\begin{tabular}{|c|c|c|}
\hline Anterior (mm) & Lateral $(\mathrm{mm})$ & Ventral $(\mathrm{mm})$ \\
\hline-6.7 & \pm 1.5 & $-3.3,-3.7$ \\
\hline-6.0 & \pm 1.8 & $-3.3,-3.7$ \\
\hline-6.0 & \pm 1.8 & -3.7 \\
\hline-5.4 & \pm 3.0 & $-3.3,-3.7^{*}$ \\
\hline-5.4 & \pm 1.4 & $-3.3 *$ \\
\hline-4.8 & \pm 1.8 & -3.3 \\
\hline-4.8 & \pm 3.8 & $-3.5^{*}$ \\
\hline-4.2 & \pm 5.0 & $-4.7^{*},-7.5^{*}$ \\
\hline-4.2 & \pm 2.8 & 3.5 \\
\hline-3.5 & \pm 4.5 & $-4.5 *,-7.0^{*}$ \\
\hline-2.9 & \pm 4.8 & $-4.5 *,-7.0^{*}$ \\
\hline
\end{tabular}

All coordinates are given with reference to stereotaxic zero according to the Paxinos and Watson (1986) rat brain atlas. The injected volume was 0.125 microliters at all sites except those marked with an $*$ were 0.2 microliters.

responding to the preexposed CS than to the novel CS would demonstrate latent inhibition.

\section{Materials and Methods}

Subjects. The subjects were 32 male Long-Evans rats (Charles River Laboratories, Raleigh, NC), 300-325 gm at the beginning of the experiment. $\Lambda$ fter $21 \mathrm{~d}$ of postoperative recovery, the rats were placed on a restricted feeding regimen and gradually reduced to $85 \%$ of their postoperative ad libitum weights. The rats were maintained at those weights for the remainder of the experiment.

Surgery. Bilateral lesions of the hippocampal formation were made by injection of a solution of ibotenic acid and $N$-methyl-D-aspartate (NMDA), both agonists with differing affinities for subtypes of ionotropic and metabatropic glutamate receptors. Prior work in our laboratories has shown that the combination of neurotoxins produces more reliable lesions than the use of ibotenic acid alone. The neurotoxins were obtained from Regis Chemical Company, while rats were anaesthetized with Nembutal $(50 \mathrm{mg} / \mathrm{kg}$ ). The stereotaxic coordinates were adopted from the Paxinos and Watson (1986) atlas, as shown in Table 1. Ibotenic acid $(10 \mathrm{mg} / \mathrm{ml})$ and NMDA $(10 \mathrm{mg} / \mathrm{ml})$ were dissolved in phosphate buffered saline, and administered slowly in a $0.2 \mu \mathrm{l}$ volume at each site through a 30 gauge stainless steel cannula attached to a 1.0 $\mu l$ Hamilton microsyringe. The cannula was left in place at each injection site for 3 min. The rats in the control group underwent the same surgical procedures with the exception that vehicle injections were administered.

Apparatus. The apparatus consisted of eight individual chambers, each $22.9 \times 20.3 \times 20.3 \mathrm{~cm}$, with aluminum front and back walls, clear acrylic sides and top, and a grid floor $(0.48 \mathrm{~cm}$ stainless steel rods spaced $1.9 \mathrm{~cm}$ apart). A dimly illuminated food cup was recessed in the center of one end wall; a $6 \mathrm{~W}$ jeweled panel light, which was the source of one visual $\mathrm{CS}$, was located $5 \mathrm{~cm}$ above the opening to that recess. Each experimental chamber was enclosed in a sound-resistant shell with acrylic windows for viewing the rats. A $6 \mathrm{~W}$ normally off house light, which served as the other visual CS, was mounted on the inside wall of the shell, $25 \mathrm{~cm}$ above and behind the experimental chamber, aligned with the end wall that contained the food cup. A speaker, used to presenti the auditory CS used in Experiment 2, was mounted next to the house light. Ventilation fans provided masking noise ( 70 $\mathrm{dB}$ ), and a $6 \mathrm{~W}$ lamp behind a red lens opposite the house light provided continuous dim background illumination. Two low-light television cameras were mounted $2.1 \mathrm{~m}$ from the experimental chambers so each could include four chambers in its field of view. Videocassette recorders were programmed to record behaviors that occurred during the $10 \mathrm{sec}$ intervals before, during, and after CS presentations. Because conditioned hehaviors were seldom observed prior to CS presentations, pre-CS responding was not reported in these experiments.

Behavioral observation procedures. All observations were made from videotapes, and paced by auditory signals recorded on the tapes. For each rat, observations were made at $1.25 \mathrm{sec}$ intervals during the 5 sec period immediately prior to CS presentations and during CS pre- sentations. At each observation, one and only one behavior was recorded.

Two broad categories of behavior were reported. Rear behavior (standing on the hind legs, with both front legs off the floor, but not grooming) occurs initially as an unconditioned, orienting response to visual CSs like those used in these experiments, but also is potentiated by pairings of the CS with the unconditioned stimulus (US). Food-cup behavior, which included standing motionless in front of the recessed food cup, with the head or nose within the recessed area, and head-jerk behavior (short, rapid, horizontal and/or vertical movements of the head) oriented toward the food cup, initially occurs in response to delivery of the food US, but is rapidly acquired to CSs paired with food Because previous data (e.g., Holland, 1977, 1984) showed that rear behavior occurs primarily during the first $5 \mathrm{sec}$ period of a $10 \mathrm{sec} C S$, and food-cup behavior occurs primarily during the last $5 \mathrm{sec}$ of those CSs, we report the frequencies of rear behavior during the first half of the 10 sec CS intervals, and of food-cup behavior during the last half of the CS intervals. It is worth noting that because these two behaviors occur primarily at different times, there is little competition between them in performance.

The index of behavioral frequency used was percentage total behavior, obtained by dividing the frequency of the target behavior in any observation interval by the total number of observations made in that interval. Note that because the number of observations was constant within each observation interval, this measure is an absolute frequency measure, not a relative one. A single primary observer $(\mathrm{PCH})$ scored all of the behavioral data reported here. To assess objectivity, a second observer also scored the data from several of the test sessions. The two observers agreed on $94 \%$ of 1280 joint observations. Neither observer was aware of the rats' lesion condition when the data were scored; in addition, in the acquisition test sessions, neither observer knew which CS had been preexposed.

Training procedures. First, in each of five daily $64 \mathrm{~min}$ preexposure sessions, the rats received eight $10 \mathrm{sec}$ presentations of either the panel light or the house light stimulus (counterbalanced within each lesion condition). Next, the rats were trained to eat from the food cups. Sixteen deliveries of two $45 \mathrm{mg}$ food pellets (which served as the US throughout these experiments) were given at random times within a single $64 \mathrm{~min}$ session. Finally, in each of 10 daily $64 \mathrm{~min}$ acquisition test sessions, all rats received four $10 \mathrm{sec}$ presentations of the house light, followed immediately by the food US, and four $10 \mathrm{sec}$ presentations of the panel light, also followed immediately by the food US. These trials were presented in randomly intermixed orders, with variable intertrial intervals that averaged 8 min.

Experiment 1 was conducted in two identical replications, with each of the lesion and CS counterbalancing conditions represented in each replication.

Histology. After completion of behavioral testing, the rats were deeply anaesthetized with chloral hydrate (30\%) and perfused transcardially with $0.9 \%$ saline followed by $10 \%$ formalin. Brains were removed and stored in $10 \%$ formalin for 1 week. The brains were then sectioned ( 50 $\mu \mathrm{m})$ in a cryostat, mounted on slides, and Nissl stained. Coronal sections were taken through the dorsal hippocampus, and horizontal sections were taken through the ventral hippocampus. Histological examination was performed with the aid of the Paxinos and Watson (1986) atlas using an Olympus BH-2 microscope.

Data analysis. In the statistical analyses of the behavioral measures, we used two-tailed, distribution-free statistics. We adopted the $0.05 \mathrm{lev}$ el of significance.

\section{Results and Discussion}

\section{Histology}

The amount of damage to the cell fields in hippocampus (CA1 and $\mathrm{CA} 2 / \mathrm{CA} 3$ ) and to the dentate gyrus was estimated through the dorsal/ventral extent of these structures. The cell field was rated as spared even when neurons appeared sparser than normal. This analysis revealed that six lesioned rats had less than $50 \%$ damage bilaterally to the entire dorsal/ventral extent of the hippocampus/dentate gyrus. The behavioral data for those rats were discarded. The remaining rats $(n=12)$ had greater than $50 \%$ damage bilaterally. On the average, neurons in approximately $75 \%$ of the hippocampus/dentate gyrus were removed by the neu- 

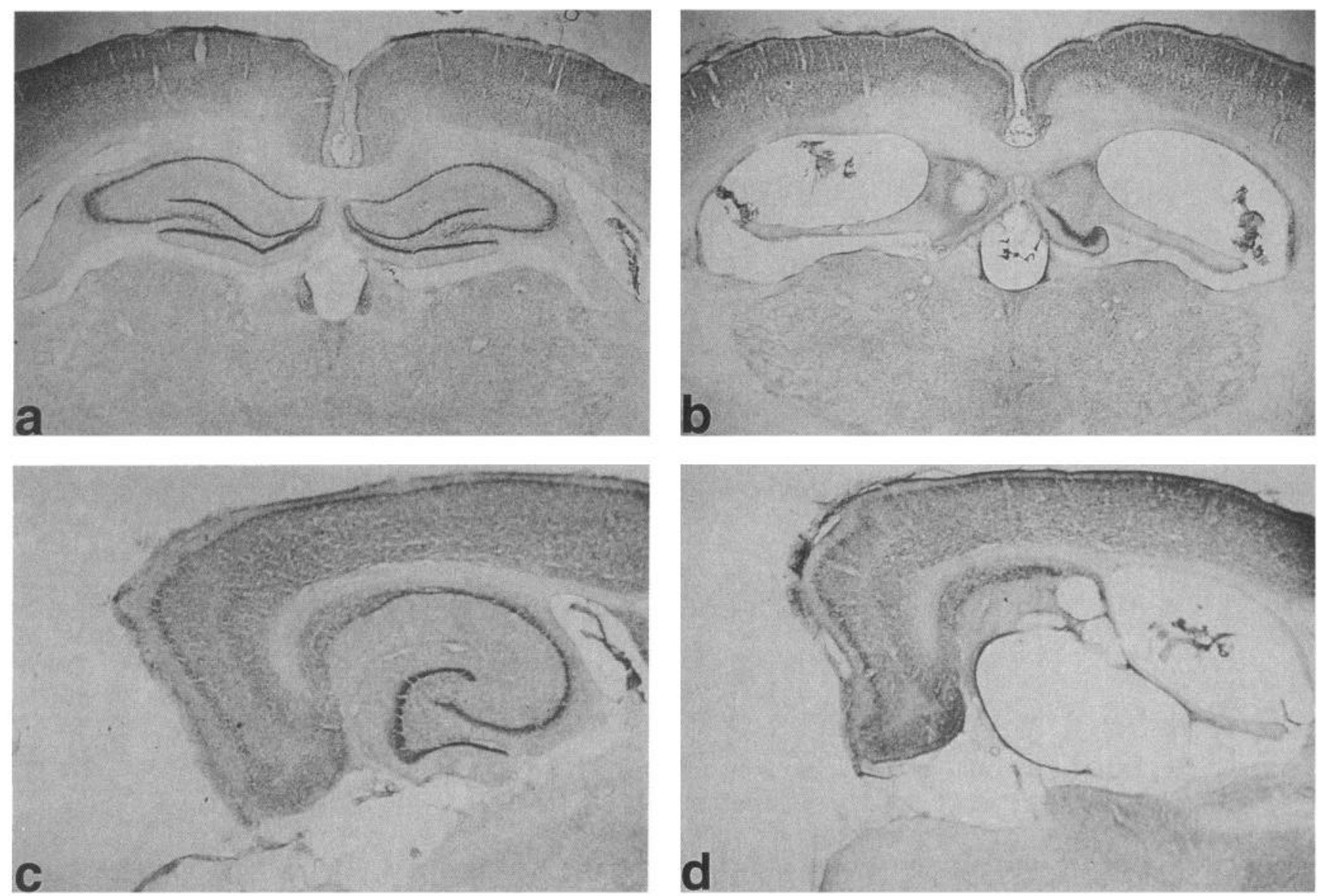

Figure 1. Photomicrographs show Nissl-stained sections taken in the coronal plane through the dorsal hippocampus $(a-b)$ and horizontal sections of the ventral hippocampus $(c-d)$. The upper and lower panels on the left $(a$ and $c$ ) are from a control brain. The neurotoxic lesion brain in the panels on the right shows extensive loss of neurons in the dorsal hippocampus, with some sparing of dentate granule cells $(b)$ and complete removal of the hippocampus/dentate gyrus cells in the ventral hippocampus sparing the subiculum and adjacent cortex $(d)$.

rotoxin. Among these lesioned rats, three had unilateral damage that extended into the subiculum. Two rats in the control group had damage to the CA1 field, which appeared to have been caused by mechanical disruption during the injection procedures. The behavioral data from these rats were also excluded from the analysis, leaving 12 rats in the vehicle control group. Figure 1 shows the extent of hippocampal damage in a rat in the lesioned group compared to a control rat. In this case, there was no significant damage to the subiculum on either side of the brain.

\section{Behavioral data}

Figure 2 shows the performance of unconditioned rear behavior during the visual cue in the preexposure phase, in which one of the two visual cues was presented without food reinforcement. Although lesioned rats appeared to show more rear behavior than control rats, those differences were not reliable, either over all five sessions, or in any individual session, Mann-Whitney $U \mathrm{~s}(12,12)>46.5, p \mathrm{~s}>0.10$.

The top panel of Figure 3 shows conditioned food-cup responding to the two CSs in the acquisition test phase. The control rats showed latent inhibition: the level of conditioning to the initially novel cue was greater than that to the preexposed cue, Wilcoxon $T(12)=3$. In contrast, lesioned rats showed equivalent acquisition to the two CSs, $T(12)=21$. Furthermore, the magnitude of the latent inhibition effect (the difference be- tween responding during the novel cue and that during the preexposed cue) was reliably greater in control rats than in the lesioned rats, $U(12,12)=34.5$. Thus, latent inhibition, the loss of associability of a stimulus as a consequence of simple nonreinforced preexposure, was apparently eliminated by the hippocampal lesions.

A similar conclusion is suggested by the performance of conditioned rear behavior (Fig. 3, bottom). Over all eight conditioning sessions, the control rats showed reliably greater responding during the novel cue than during the preexposed stimulus, $T(12)=$ 5.5 , whereas the lesioned rats did not, $T(12)=48$. Likewise, the difference between responding during the novel and preexposed cues was significantly greater in the control than in the lesioned rats, $U(12,12)=23.5$. It should be noted that in the first two conditioning sessions, rear behavior was more frequent during the novel than during the preexposed stimulus in both lesioned, $T(12)$ $=9$, and control, $T(12)=12$, rats. It is unlikely that this difference reflects latent inhibition in the lesioned rats, however. Recall that rear behavior occurs first as an unconditioned orienting response to visual stimuli (see Fig. 2), but is potentiated when those CSs are paired with food. Thus, it is likely that rear behavior in the first two conditioning sessions reflected mostly unconditioned rear behavior, rather than conditioned rear behavior. In summary then, with both measures of learning, conditioned food cup and conditioned rear behavior, hippocampal lesions disrupted latent 


\section{REAR BEHAVIOR}

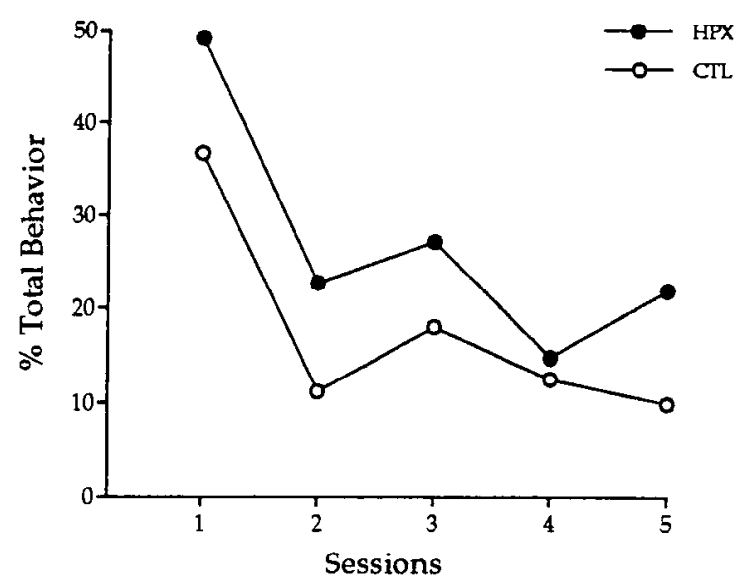

Figure 2. Rear behavior during the visual cue in the preexposure phase of Experiment 1. HPX, rats with hippocampal lesions; $C T L$, control rats.

inhibition, indicating that hippocampal circuitry is involved in decrements in CS processing.

This observation is consistent with the results from a number of investigations that entailed large, nonspecific lesions of hippocampus (e.g., Solomon and Moore, 1975; Kaye and Pearce, 1987a,b). The deficit in latent inhibition observed with neurotoxic hippocampal lesions in the present study was also recently reproduced in our laboratories by immunotoxic lesions of the septal-hippocampal cholinergic projection (Baxter and Gallagher, unpublished observations). However, it conflicts with the results of Honey and Gond (1993; Fxperiment 2), who found that neurotoxic lesions of hippocampus failed to disrupt latent inhibition. In their study, appetitive conditioning of rats was equally slowed after CS preexposure, regardless of lesion condition. One possible account for the discrepancy between Honey and Good's (1993) data and ours is that the deficit we observed is the result of damage to brain regions that were intact in their rats. In the absence of complete descriptions of histological outcomes in their study, however, it is difficult to speculate on this possibility. But it is worth pointing out that we found little evidence for damage to the subiculum, which Honey and Good (1993) suggested might be responsible for the disruptions in latent inhibition observed with larger, nonspecific hippocampal lesions.

Several procedural differences may also have contributed to this discrepancy between our results and those of Honey and Good (1993). First, whereas Honey and Good's (1993) experiments used auditory cues, ours involved visual cues. Second, it is possible that our within-subject design and large number of subjects was more sensitive to lesion effects than their betweensubject design, which used only three to five subjects in each condition (the within-subject manipulation in Experiment 3 in Honey and Good's, 1993, report, involved a contextual variable and so is not comparable to our procedures). Alternately, it might be argued that our use of a within-subjects design permitted lesion-produced differences in generalization to contribute to our findings. Some investigators have found that hippocampal lesions enhance generalization between cues of the same stimulus modality (e.g., Solomon and Moore, 1975). Consequently, the failure of our lesioned rats to show differential conditioning of the preexposed and novel cues may have reflected
FOOD CUP

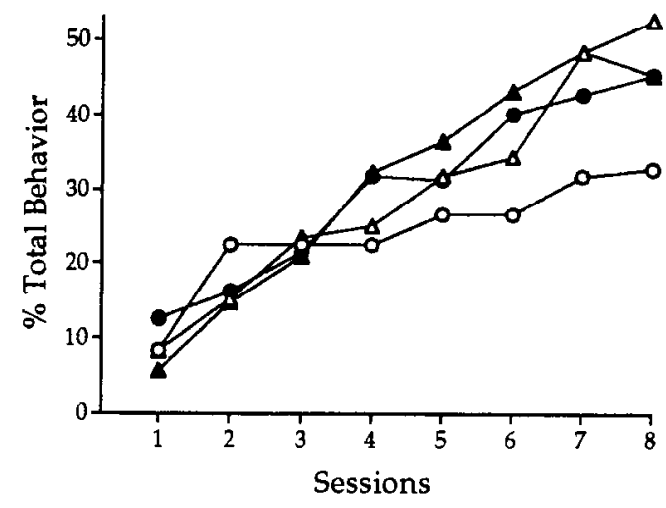

$\rightarrow$ Cr.p

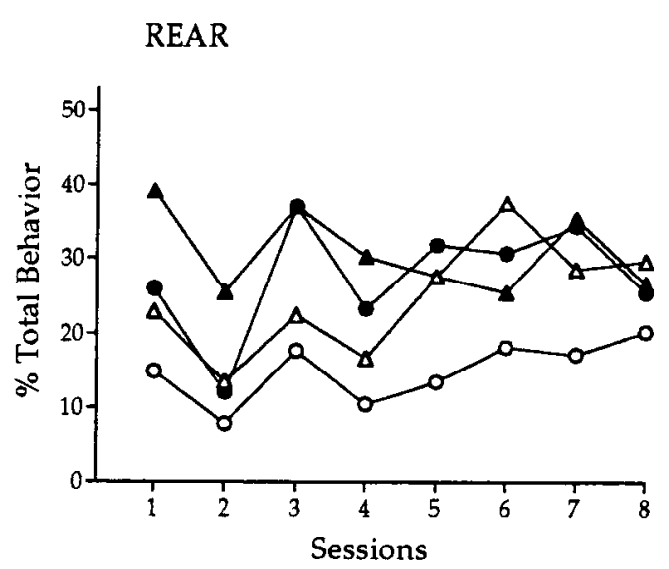

Figure 3. Food cup (top) and rear (bottom) behaviors during the conditioning phase of Experiment 1. $H P X$, rats with hippocampal lcsions; $C T L$, control rats.

enhanced generalization between them rather than reduced latent inhibition of the preexposed cue.

Two aspects of our data make this latter account unlikely, however. First, it is clear that the lesioned rats discriminated the two visual cues in the first two conditioning sessions, because they showed greater unconditioned rear behavior to the novel than to the preexposed stimulus. Second, if latent inhibition were unimpaired by the hippocampal lesions, then generalization between the two cues should have resulted in slower conditioning to the novel cue than in control rats, rather than only faster conditioning to the preexposed cue, as is evident in Figure 2. Thus, it seems simpler to attribute the lesion effect observed here to interference with decremental attentional processes that normally occur when a cue is repeatedly presented by itself prior to conditioning. Nevertheless, in Experiment 2 we examined the effects of hippocampal lesions on decrements in CS processing in another, between-subjects, experimental design, which was not subject to these criticisms.

\section{Experiment 2}

The procedure used (outlined in Table 2) was first described by Wilson et al. (1992), and was later used by Holland and Gallagher (1993a) and Chiba et al., (1994, 1995) in their investigations of the roles of amygdala $\mathrm{CN}$ and substantia innominata/ nBM in conditioning. In that procedure, both decreases and increases in CS processing are typically observed in intact rats, in 


\begin{tabular}{|c|c|c|c|}
\hline $\begin{array}{l}\text { Training condition } \\
\text { (Groups) }\end{array}$ & $\begin{array}{l}\text { Phase 1: } \\
\text { consistent } \\
\text { light (L)-tone (T) } \\
\text { relationship }\end{array}$ & $\begin{array}{l}\text { Phase } 2 \text { : } \\
\text { experimental change in } \\
\text { light }(L) \text {-tone }(T) \\
\text { relationship }\end{array}$ & $\begin{array}{l}\text { Phase } 3 \text { : } \\
\text { test of } \\
\text { learning } \\
\text { to light }(\mathrm{L})\end{array}$ \\
\hline $\begin{array}{l}\text { Consistent } \\
\text { (CTL-C/HPX-C) }\end{array}$ & $\mathrm{L} \rightarrow \mathrm{T} \rightarrow$ food $; \mathrm{L} \rightarrow \mathrm{T}$ & $\mathrm{L} \rightarrow \mathrm{T} \rightarrow$ food $; \mathrm{L} \rightarrow \mathrm{T}$ & $L \rightarrow$ food \\
\hline $\begin{array}{l}\text { Shifted } \\
\text { (CTL-S/HPX-S) }\end{array}$ & $\mathrm{L} \rightarrow \mathrm{T} \rightarrow$ food $\mathrm{L} \rightarrow \mathrm{T}$ & $\mathrm{L} \rightarrow \mathrm{T} \rightarrow$ food; $\mathrm{L}$ & $\mathrm{L} \rightarrow$ food \\
\hline
\end{tabular}

CTL, control. HPX, hippocampal lesion. L, light conditioned stimulus (CS). T, tone CS. fd, food unconditioned stimulus (US). $\rightarrow$ signifies serial relation.

separate phases. Thus, in addition to examining the effects of hippocampal lesions in reductions in CS processing, Experiment 2 investigated the effects of those lesions on increases in CS processing.

In the first Phase of Experiment 2, all subjects were first exposed to a serial conditioning schedule in which a light-thentone sequence was followed by a food US half of the time (light $\rightarrow$ tone $\rightarrow$ food and light $\rightarrow$ tone $\rightarrow$ nothing trials). Thus, although the light and tone were both followed by the food US on a $50 \%$ reinforcement schedule, the light was consistently followed by the tone on all trials. As a consequence of this schedule, the tone should acquire substantial conditioned responding, but, because of its relatively poor temporal relation with food, the light is likely to acquire only minimal conditioned responding. Most important for the purpose of this study, as serial conditioning proceeds, the light comes to accurately predict the tone, and attention to the light is reduced (Wilson et al., 1992).

In the second phase of Experiment 2, attention to the light was manipulated by altering the light's relationship to the tone in one group of lesioned rats (group HPX-S) and one group of unlesioned rats (group CTL-S). Those rats continued to receive light $\rightarrow$ tone $\rightarrow$ food trials, but the light $\rightarrow$ tone $\rightarrow$ nothing trials of phase 1 were replaced by light-alone trials. Thus, although the phase 1 light-food relation was maintained, the light no longer predicted the tone reliably. As a result of this change in the light's predictive value, attentional processing of the light CS should be enhanced in these two groups, according to Pearce and Hall's (1980) model. The comparison groups of lesioned (group HPXC) and unlesioned (group CTL-C) rats continued to receive the phase 1 procedures, light $\rightarrow$ tone $\rightarrow$ food and light $\rightarrow$ tone $\rightarrow$ nothing trials, thus providing them with extended training with the procedure intended to reduce attention to the light.

Attention to the light was then assessed in all four groups by pairing the light directly with food in a final test phasc. Based on previous suggestions that hippocampal systems might be involved in decremental but not incremental changes in CS processing (Moore and Stickney, 1980; Holland and Gallagher, 1993a,b), we made two predictions. First, among the rats that received extended training with consistent light $\rightarrow$ tone pairings, only the intact rats should show decrements in attention to the light. Consequently, the lesioned rats in group HPX-C should show more attention to the light, and hence, more conditioning in the test phase, than the intact rats in group CTL-C. Second, the phase 2 shift in predictive accuracy of the light should increase controlled attention, and hence, enhance light-food conditioning in the final test sessions, in both intact (group CTL-S) and lesioned (group HPX-S) rats. This prediction is based on previous studies indicating that the incremental function is served by other neural circuitry (Holland and Gallagher, 1993a,b; Chiba et al., 1995).

\section{Materials and Methods}

Surgical and histological procedures. The surgical and histological procedures were identical to those of Experiment 1.

Subjects, apparatus, and behavioral obsenation procedures. The subjects were 52 ( 25 lesion and 27 control) male Long-Evans rats (Charles River Laboratories, Raleigh, NC), 300-325 gm at the beginning of the experiment. They were maintained in the same manner as the rats in Experiment 1. The apparatus and behavioral observation procedures were the same as those used in Experiment 1.

Training procedures. The rats were first trained to eat from the food cups, as in Experiment 1 . Then, all rats received 10 daily 64 min phase 1 conditioning sessions. In each of those sessions, the rats received four reinforced and four nonreinforced presentations of a light-tone serial compound CS, randomly intermixed, with variable intertrial intervals that averaged $8 \mathrm{~min}$. The serial compound comprised a $10 \mathrm{sec}$ illumination of the panel light, followed immediately by a $10 \mathrm{sec}$ presentation of a $10 \mathrm{sec}, 78 \mathrm{~dB}, 1500 \mathrm{~Hz}$ tone. On reinforced trials, the tone was followed immediately by two $45 \mathrm{mg}$ food pellets.

In phase 2, the lesioned rats in group HPX-C $(n=12)$ and the unlesioned rats in group $C T L-C(n=16)$ received 10 daily sessions identical to those given in phase 1 . In each of the 10 daily 64 min phase 2 sessions, the lesioned rats in group HPX-S $(n=10)$ and the unlesioned rats in group CTL-S $(n=11)$ received four light $\rightarrow$ tone $\rightarrow$ food trials like those given in phase 1 , intermixed with four $10 \mathrm{sec}$ presentations of the panel light alone. As in phase 1, the intertrial intervals were variable and averaged $8 \mathrm{~min}$.

All rats then received five 64 min daily test sessions. In each of those sessions, eight $10 \mathrm{sec}$ illuminations of the panel light were each followed by the two-pellet food US. Again, the intertrial intervals were variable and averaged $8 \mathrm{~min}$. The experiment was conducted in three replications

\section{Results}

\section{Histology}

Twenty-two rats that had received neurotoxic injections had lesions that produced greater than $50 \%$ damage to each hippocampus/dentate gyrus. Behavioral data from the other three lesioned rats were discarded. The extent of the lesions was comparable for the two training conditions (mean of $74 \%$ removal of the target structures in group HPX-C, $n=12$, and $76 \%$ damage in group HPX-S, $n-10$ ). Five of these rats had a small amount of unilateral damage to the subiculum; three of these were in group HPX-C and two were in group HPX-S. One additional rat in group HPX-C had a small amount of bilateral damage in the subiculum. No extraneous damage was detected in the vehicleinjected brains, yielding 11 rats in group CTL-S and 16 in group CTL-C. 
Table 3. Data from phases 1 and 2 of experiment 2

\begin{tabular}{|c|c|c|c|c|c|c|}
\hline \multirow[b]{2}{*}{ Group } & \multicolumn{3}{|c|}{$\begin{array}{l}\text { Final two sessions } \\
\text { of phase } 1\end{array}$} & \multicolumn{3}{|c|}{$\begin{array}{l}\text { Final two sessions } \\
\text { of phase } 2\end{array}$} \\
\hline & $\begin{array}{l}\text { Light } \\
\text { (rear) }\end{array}$ & $\begin{array}{l}\text { Light } \\
\text { (fc) }\end{array}$ & $\begin{array}{l}\text { Tone } \\
\text { (fc) }\end{array}$ & $\begin{array}{l}\text { Light } \\
\text { (rear) }\end{array}$ & $\begin{array}{l}\text { Light } \\
\text { (fc) }\end{array}$ & $\begin{array}{l}\text { Tone } \\
\text { (fc) }\end{array}$ \\
\hline HPX-S & 24.3 & 10.3 & 55.6 & 19.5 & 16.0 & 62.4 \\
\hline HPX-C & 23.2 & 11.2 & 55.3 & 21.4 & 18.5 & 60.0 \\
\hline CTL-S & 24.4 & 11.9 & 58.4 & 16.6 & 15.5 & 60.4 \\
\hline CIL-C & 26.3 & 9.1 & 56.2 & 18.6 & 16.8 & 59.1 \\
\hline
\end{tabular}

Entries are percentages of total behavior (see text). fc, food cup behavior.

\section{Behavioral results}

Table 3 shows responding over the final two sessions of phase 1 (left columns) and phase 2 (right columns). In all four groups, the tone CS controlled substantial food cup CRs, but the visual CS (which was more temporally remote from the US) controlled only small amounts of rear and food cup behaviors. There were no differences among the groups in responding during either the light or the tone CSs, either in these sessions or in any other sessions in phases 1 and 2, Mann-Whitney $U_{\mathrm{s}}>61$. Thus, the hippocampal lesions did not affect the acquisition or maintenance of conditioning in phases 1 and 2 .

Figure 4 shows the primary data of Experiment 2, the acquisition of food-cup behavior during the light in the light-food test sessions, during which the light-food temporal interval was made conducive to conditioning (rear behavior, not shown, exhibited comparable patterns of results). Conditioning was rapid; compare performance during the light $\mathrm{CS}$ on the first conditioning test trial $(\mathrm{P})$ with performance over the first conditioning session. Nevertheless, the amount of conditioned responding observed in the test phase varied as a function of both phase 2 treatment and lesion condition.

First, hippocampal lesions apparently interfered with reductions in CS processing in the rats that received extended training with consistent light $\rightarrow$ tone pairings (group HPX-C). The lesioned rats in that group showed more conditioned food-cup behavior throughout the test phase than the intact rats that received the same treatment (group CTL-C), $U(16,12)=35.5$. Nlthough it might be claimed that this superiority reflects gen erally enhanced conditioning in the lesioned rats rather than the absence of normally occurring decrements in CS associability, the lack of any lesion-induced differences in CRs throughout phases 1 and 2 (Table 3 ) refutes that interpretation.

Second, shifting the predictive value of the light in phase 2 enhanced attention to the light in both intact and lesioned rats. Rats that received phase 2 shift training designed to reestablish or enhance attention to the light (groups CTL-S and HPX-S), showed more food-cup responding than the rats in the corresponding unshifted groups, CTL-C, $U(16,11)=38.5$, and HPX$\mathrm{C}, U(12,10)=31$. Thus, the hippocampal lesions apparently did not eliminate increments in attention to a CS produced by shifts in its predictive value.

Performance of lesioned rats in the consistent, unshifted condition (group HPX-C) was very similar to that of unlesioned rats in the shift (group HPX-S) condition, $U(12,11)=64.5$. This finding may indicate that the shift manipulation permitted the recovery of attention in intact rats to the same level as would have existed in the absence of a decremental processing mechanism, consistent with the predictions of the Pearce-Hall (1980)

\section{FOOD CUP BEHAVIOR}

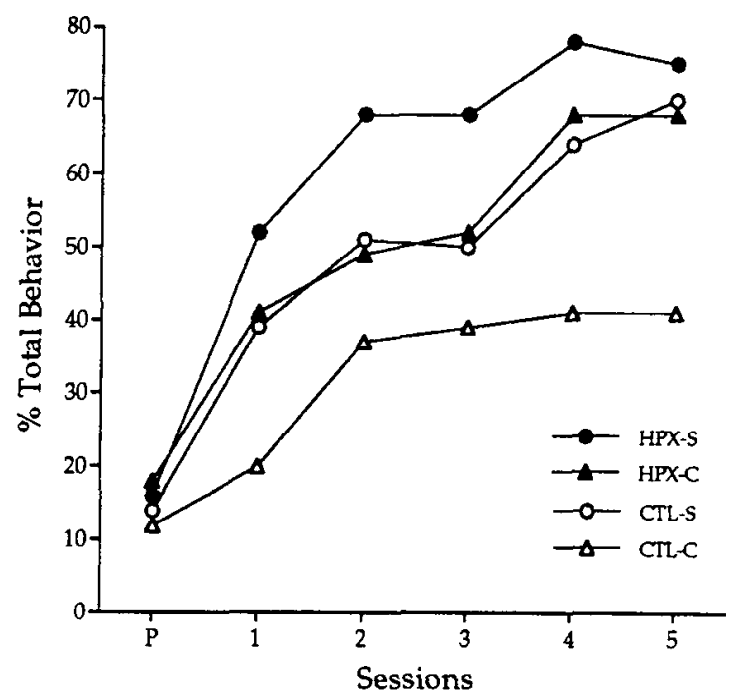

Figure 4. Food-cup behavior during the test sessions of Experiment 2. $H P X$, rats with hippocampal lesions; $C T L$, control rats; $S$, rats for which the training contingencies were shifted in phase $2 ; C$, rats for which the phase 1 training contingencies were continued in phase 2 .

model. If so, then the superior performance of shifted, lesioned rats (group HPX-S) relative to that of both group HPX-C (noted earlier) and group CTL-S, $U(11,10)=30.5$, might suggest that shift-induced enhancement of attention proceeds regardless of whether attention has been previously decremented. Alternately, the comparable performance of groups CLT-S and HPX-C may have reflected both an incomplete recovery of attention after the shift in group CTL-S and an incomplete effect of the hippocampal lesions on decremental processes in group HPX-C. Either way, these data indicate that hippocampal lesions interfere with decremental, but not incremental changes in CS processing.

\section{General Discussion}

The results of two experiments showed that neurotoxic lesions of hippocampus interfered with decremental changes in the processing of CSs. In Experiment 1, lesioned rats failed to show reduced latent inhibition, the retardation of learning to a $C S$ after extensive preexposure to that stimulus alone. In Experiment 2, lesioned rats failed to show retarded learning to a CS that had been extensively trained as a consistent predictor of another event. Although inconsistent with Honey and Good's (1993) failure to find an effect of neurotoxic hippocampal lesions on latent inhibition, these results are consistent with the results of other studies of the effects of less specific hippocampal lesions on latent inhibition (e.g., Solomon and Moore, 1975; Kaye and Pearce, 1987a,b), and on reductions in CS associability after extended training with consistent CS-US pairings (Kaye and Pearce, 1987a). The results of Experiment 2 also revealed that both intact and lesioned rats showed increments in attentional processing of a CS when that cue was made an unreliable predictor of subsequent events.

Other evidence from our laboratories suggests that different neural systems may be involved in these incremental and decremental changes in attentional processing of CSs (e.g., Holland and Gallagher, 1993a,b; Chiba et al., 1994; Gallagher and Hol- 
land, 1994). Neurotoxic lesions of the amygdala central nucleus (CN) and immunotoxic lesions of cholinergic neurons in the substantia innominata/nucleus basalis (SI/nBM) region each interfered with rats' performance in tasks that involve increments in controlled attentional processing according to Pearce and Hall's (1980) model. Conversely, these lesions had no effect on performance in tasks that involved decrements in CS processing (e.g., latent inhibition). Together with the results of those studies, the present findings indicate a double dissociation between incremental and decremental changes in controlled attentional processing. Whereas lesions to $\mathrm{CN}$ or $\mathrm{SL} / \mathrm{nBM}$ interfered with incremental changes but left decremental changes unaffected, hippocampal lesions interfered with decremental changes but not incremental changes.

The existence of separate neural systems mediating incremental and decremental changes in processing of CSs is informative for attempts to refine computational behavioral models of associative learning. For example, although most behavioral theories view incremental and decremental changes in CS processing as variations along a single continuum (e.g., Mackintosh, 1975; Pearce and Hall, 1980; but see Moore and Stickney, 1980), data like those presented here suggest that those processes may often act relatively independently. Consequently, the behavioral effects of a given manipulation might be the result of increases in one process or decreases in the other, or some combination of the two.

It is also worth relating these ideas to concepts of attention in cognitive neuroscience. Traditionally, attentional function has been most simply characterized as involving competition among items for limited processing capacity and filtering out of information that is irrelevant to the task at hand (e.g., Broadbent, 1958). Our findings address the filtering aspects of attention, indicating "top-down" processes, in which events are selected or discarded on the basis of their significance in predicting the occurrence of other events, rather than on any intrinsic properties of the events themselves. It is notable that in studies like ours these top-down selection processes operate in the absence of any obvious competition for limited resources: the visual CSs used were physically simple and were never presented simultaneously with other events, in contrast to the complex arrays typically used in investigations of visual attention. At the same time, it is likely that these top-down selection processes would operate under conditions of stimulus competition as well; indeed, it is just those circumstances that modern theories of selective attention in associative learning were designed to encompass. Finally, our proposal of separate incremental and decremental processing mechanisms subserved by amygdalar and hippocampal systems (respectively), is consistent with the developing notion that attention reflects the interaction of many neural mechanisms to resolve competition for attentional processing and the control of behavior (Desimone and Duncan, 1995).

\section{References}

Berger TW, Orr WB (1985) Hippocampectomy disrupts topography of conditioned nictitating membrane responses during reversal learning. Behav Neurosci 99:35-45.

Berger TW, Alger BE, Thompson RF (1976) Neuronal substrate of classical conditioning in the hippocampus. Science 192:483-485.

Berger TW, Orr WB (1983) Hippocampectomy selectively disrupts dis crimination reversal conditioning of the rabbit nictitating membrane response. Behav Brain Res 8:49-68.
Best MR, Best PJ (1976) The effects of latent inhibition on hippocampal unit activity in the rat. Exp Neurol 51:546-573.

Broadbent DE (1958) Perception and communication. London: Pergamon.

Chiba AA, Han JS, Holland PC, Gallagher M (1994) Substantia innominata lesions impair incremental attentional processing in a Pavlovian conditioning paradigm. Soc Neurosci Abstr 20:1215.

Chiba AA, Bucci DJ, Holland PC, Gallaher M (1995) Basal forebrain cholinergic lesions disrupt increments but not decrements in conditioned stimullus processing. J Neurosci 15:7315-7322.

Desimone R, Duncan J (1995) Neural mechanisms of selective visual attention. Annu Rev Neurosci 18:193-222.

Dickenson A, Mackintosh NJ (1979) Reinforcer specificity in the enhancement of conditioning by posttrial surprise. J Exp Psychol Anim Behav Process 5:162-177.

Douglas RJ (1967) The hippocampus and behavior. Psychol Bull 67: $416-442$.

Gallagher M, Holland PC (1994) The amygdala complex: multiples roles in associative learning and attention. Proc Natl Acad Sci USA 91:11771-11776.

Gluck MA, Meyers CE (1993) Hippocampal mediation of stimulus representation: a computational theory. Hippocampus 3:491-516.

IIolland PC (1977) Conditioned stimulus as a determinant of the form of the Pavlovian conditioned response. J Exp Psychol Anim Behav Process 3:77-104.

Holland PC (1984) The origins of Pavlovian conditioned behavior. In: The psychology of learning and motivation, Vol 18 (Bower G, ed), pp 129-173. Englewood Cliffs, NJ: Prentice-Hall.

Holland PC, Gallagher M (1993a) Amygdala central nucleus lesions disrupt increments, but not decrements, in conditioned stimulus processing. Behav Neurosci 107:246-253.

Holland PC, Gallagher M (1993b) The effects of amygdala central nucleus lesions on blocking and unblocking. Behav Neurosci 107: 235-245.

Holland PC, Gallagher M (1995) Attention and learning. In: Learning and memory (Elsner N, Menzel R, eds). Stuttgart: Thieme.

Honey RC, Good M (1993) Selective hippocampal lesions abolish the contextual specificity of latent inhibition and conditioning. Behav Neurosci 107:23-33.

Kaye II, Pearce JM (1987a) IIippocampal lesions attenuate latent inhibition and the decline of the orienting response in rats. Q J Exp Psychol 39B:107-125.

Kaye H, Pearce JM (1987b) Hippocampal lesions attenuate latent inhibition of a CS and of a neutral stimulus. Psychobiology 15:293-299.

Mackintosh NJ (1975) A thoery of attention: variations in the associability of stimuli with reinforcement. Psychol Rev 82:276-298.

Moore JW, Stickney KJ (1980) Formation of attentional-associative networks in real time: role of the hippocampus and implications for conditioning. Physiol Psychol 8:207-217.

Olds J, Disterhoft JF, Segal M, Kornblith CL, Hirsch R (1972) Learning centers of the rat brain mapped by measuring latencies of conditioned unit responses. J Neurophysiol 35:202-219.

Paxinos G, Watson C (1986) The rat brain in stereotaxic coordinates. New York: Academic.

Pearce JM, Hall G (1980) A model for Pavlovian learning: variations in the effectiveness of conditioned but not of unconditioned stimuli. Psychol Rev 106:532-552.

Rickert EJ, Bennett TL, Lane P, French J (1978) Hippocampectomy and the attenuation of blocking. Behav Biol 22:147-160.

Schmajuk NA, DiCarlo JJ (1991) A neural netowrk approach to hippocampal function in classical conditioning. Behav Neurosci 105:82-110.

Schmajuk NA, Moore JW (1988) The hippocampus and the classically conditioned nictitating membrane response: a real-time attentionalassociative model. Psychobiology 46:20-35.

Schmaltz LW, Theios J (1972) Acquisition and extinction of a classically conditioned response in hippocampectomized rabbits (Oryctolagus cuniculus). J Comp Physiol Psychol 79:328-333.

Segal M, Disterhoft IF, Olds M (1972) Hippocampal unit activity during classical aversive and appetitive conditioning. Science 175:792-794.

Solomon P, Moore JW (1975) Latent inhibition and stimulus generalization of the classically conditioned nictitating membrane response in rabbits (Oryctolagus cuniculus) following dorsal hippocampal ablation. J Comp Physiol Psychol 89:1192-1203.

Wilson PN, Boumphrey P, Pearce JM (1992) Restoration of the orienting response to a light by a change in its predictive accuracy. $Q$ J Exp Psychol 44B:17-36. 